\title{
ON THE DISTRIBUTION OF VALUES OF BOUNDED ANALYTIC FUNCTIONS $\dagger$
}

\author{
BY \\ WLADIMIR SEIDEL $\ddagger$
}

1. Let $f(z)$ be a regular analytic function in the unit circle $|z|<1$. Consider any point $P$ of the periphery. Adopting a terminology due to W. Gross§ we may associate with $P$ three sets of points:

(i) The cluster set $C(P)$ of $f(z)$ in $P$. This is defined as the set of all those values $\alpha$ which $f(z)$ approaches on a sequence of points of the unit circle $|z|<1$ converging toward $P$.

(ii) The range of values $R(P)$ of $f(z)$ in $P$. A value $\alpha$ belongs to the set $R(P)$ if, and only if, $f(z)$ assumes the value $\alpha$ in every neighborhood of the point $P$.

(iii) The convergence set $\Gamma(P)$ of $f(z)$ in $P$. The set $\Gamma(P)$ consists of all those values $\alpha$ which $f(z)$ approaches on a Jordan arc lying, except for one end point, in the interior of the unit circle $|z|<1$ and terminating in the point $P$.

In case that the function $f(z)$ under consideration is bounded: $|f(z)|<M$ in $|z|<1$, where $M$ is some positive constant, from well known theorems one obtains at once additional information concerning the set $\Gamma(P)$. According to a theorem of Fatou\| the limit

$$
\lim _{r \rightarrow 1} f\left(r e^{i \theta}\right)=f^{*}\left(e^{i \theta}\right) \quad\left(z=r e^{i \theta}\right)
$$

exists for all values of $\theta$ in the interval $0 \leqq \theta \leqq 2 \pi$ save perhaps for a set of measure zero of values of $\theta$. The function $f^{*}\left(e^{i \theta}\right)$ will henceforth be denoted by us as the boundary function of $f(z)$. In our terminology, Fatou's theorem may be stated in the following form: The convergence set $\Gamma(P)$ of a bounded analytic function $f(z)$ in the unit circle $|z|<1$ contains at least one point for almost all points $P$ of the circumference $|z|=1$.

$\dagger$ Presented to the Society, October 29, 1932; received by the editors September 27, 1932, and, in revised form, February 18, 1933. The author is indebted to Professor J. D. Tamarkin for the suggestion of basing the proofs of Theorems $2-5$ on Theorem 1, thus simplifying the original proofs. Fellow.

‡ A part of these investigations was carried out while the author was a National Research

$\S \mathrm{W}$. Gross, Über die Singularitäten analytischer Funktionen, Monatshefte für Mathematik und Physik, vol. 29 (1918), pp. 3-47. 366-368.

II P. Fatou, Séries trigonométriques et séries de Taylor, Acta Mathematica, vol. 30 (1906), pp. 
By the preceding theorem and a theorem of Lindelöf $\dagger$, moreover, it follows immediately that the convergence set $\Gamma(P)$ of a bounded analytic function $f(z)$ in the unit circle $|z|<1$ contains one and only one point for almost all points $P$ of the circumference $|z|=1$, and no convergence set $\Gamma(P)$ can contain more than one point.

As regards the sets $C(P)$ and $R(P)$, it will suffice for the present to remark that the set $R(P)$ is always contained in the set $C(P)$.

The present paper will be concerned primarily with those bounded analytic functions $f(z)$ in the unit circle $|z|<1$, for which the modulus of the boundary function $\left|f^{*}\left(e^{i \theta}\right)\right|=1$ for almost all values of $\theta$ in the interval $0 \leqq \theta \leqq 2 \pi$. Throughout this paper such functions will be called of class $(A)$. R. Nevanlinnaf was the first to point out the interest which lies in this class of functions. There exists a wide range of well known functions which belong to the class which we propose to study. Of these we shall mention the following three groups:

(i) If $a_{1}, a_{2}, \cdots, a_{m-k}$ are points interior to the unit circle, all functions of the form

$$
R(z)=e^{i \alpha} z^{k} \prod_{i=1}^{m-k} \frac{z-a_{i}}{1-\bar{a}_{i} z}
$$

where $k$ and $m$ are positive integers and $\alpha$ is any real number. These functions yield the most general $(1, m)$ conformal correspondence of the unit circle with itself.§

(ii) All infinite products of the form

$$
B(z)=\prod_{i=1}^{\infty} \frac{1-z / a_{i}}{1-\bar{a}_{i} z}\left|a_{i}\right|,
$$

where the points $a_{i}$ are interior to the unit circle and satisfy the condition

$$
\prod_{i=1}^{\infty}\left|a_{i}\right|>0 \text {. }
$$

It has been shown by W. Blaschkell that, when condition (1.3) is satisfied,

† E. Lindelöf, Sur un principe général de l'analyse, Acta Societatis Scientiarum Fennicae, vol. 46 (1915), No. 4, pp. 1-35.

$\ddagger$ R. Nevanlinna, Über beschränkte analytische Funktionen, Annales Academiae Scientiarum Fennicae, (A), vol. 32 (1929), No. 7, p. 64.

$\S$ For a detailed account of these products, cf. T. Rad6, Zur Theorie der mehrdeutigen konformen Abbildungen, Acta Litterarum ac Scientiarum Regiae Universitatis Hungaricae Francisco-Josephinae, vol. 1 (1922), pp. 55-64; as well as G. Julia, Principes Géométriques d'Analyse, part 1, Paris, 1930, pp. 54-59.

II W. Blaschke, Eine Erweiterung des Satzes von Vitali über Folgen analytischer Funktionen, Leipziger Berichte, vol. 67 (1915), pp. 194-200; also G. Julia, loc. cit. in preceding footnote. 
the product $B(z)$ converges uniformly in every closed subregion lying wholly interior to the unit circle $|z|<1$, thus defining there an analytic function $B(z)$, which is commonly called a Blaschke product. It was shown by F. Riesz $\dagger$ that the boundary function $B^{*}\left(e^{i \theta}\right)$ of a Blaschke product $B(z)$ has the modulus 1 in almost all points of the circumference $|z|=1$. This proves that Blaschke products belong to the class of functions under consideration. In a previous paper $\ddagger$ the author showed that if the numbers $a_{i}$ in (1.2) converge to a single point $P$ of the circumference $|z|=1$, then the range of values $R(P)$ of the Blaschke product $B(z)$ consists of all the points of the unit circle $|\alpha|<1$, with the possible exception of at most one point. In this paper a further study is made of the ranges of values of Blaschke products under more general distributions of the zeros $\left\{a_{i}\right\}$.

(iii) Consider the unit circle $|w|<1$ and a set $S$ of points closed relatively to the circle $|w|<1$. On removing all points of $S$ from the unit circle $|w|<1$, at least one in general infinitely connected region $\Sigma$ is obtained. According to general existence theorems of conformal mapping $\S$ it is known that there exists an infinitely multiple-valued function $z=\phi(w)$ analytic in $\Sigma$ and assuming in it every value from the interior of the unit circle $|z|<1$ once and only once. This function is said to map $\Sigma$ conformally on the circle $|z|<1$. As will be proved in this paper, the inverse function $w=f(z)$ of the mapping function $z=\phi(w)$ belongs under appropriate restrictions on the set $S$ to the class of functions under consideration and may be represented as a linear function of a Blaschke product.

Finally, in connection with this work mention should be made of recent papers by G. Hössjer and J. L. Doob.\|

2. We begin by establishing an integral representation for all functions of class $(A)$ in the unit circle $|z|<1$. Let $f(z)$ be a function of class $(A)$ in $|z|<1$ and denote its zeros (if they exist) by $a_{1}, a_{2}, \ldots, a_{i}, \ldots$. Since $f(z)$ is bounded, by a theorem of Blaschke $\int$ its zeros satisfy the inequality $\prod_{i=1}^{\infty}\left|a_{i}\right|>0$. In accordance with the result stated in $\$ 1$, page 202, we may form the Blaschke product

† F. Riesz, Über die Randwerte einer analytischen Funktion, Mathematische Zeitschrift, vol. 18 (1923), p. 94.

For further results concerning Blaschke products cf. J. L. Walsh, Interpolation and functions analytic interior to the unit circle, these Transactions, vol. 34 (1932), pp. 523-556.

$\ddagger$ W. Seidel, On the cluster values of analytic functions, these Transactions, vol. 34 (1932), p. 17.

§ L. Bieberbach, Lehrbuch der Funktionentheorie, vol. II, 1931, chapter I, pp. 1-84.

|| G. Hössjer, Über die Randwerte beschränkter Funktionen, Acta Litterarum ac Scientiarum Regiae Universitatis Hungaricae Francisco-Josephinae, vol. 5 (1930), p. 55.

J. L. Doob, The boundary values of analytic functions, these Transactions, vol. 34 (1932), pp. 153-170.

I Leipziger Berichte, loc. cit., pp. 194-200. 


$$
B(z)=\prod_{i=1}^{\infty} \frac{1-z / a_{i}}{1-\bar{a}_{i} z}\left|a_{i}\right|,
$$

extended over the zeros $a_{i}$. Hence, by the Riesz decomposition theorem $\dagger$ if we define the function $g(z)$ by the relation $f(z)=B(z) g(z)$, we find that $g(z)$ is of class $(A)$ and different from zero in the circle $|z|<1$. Consider now the function $h(z)=\log g(z)$ which, if we select a definite branch of the logarithm, becomes single-valued and analytic in the circle $|z|<1$. Furthermore, $\Re h(z)$ $\leqq 0$ in $|z|<1$. Consequently, applying a result of Herglotz $\ddagger$ to the function $h(z)$, we obtain for it the representation

$$
h(z)=\frac{1}{2 \pi} \int_{-\pi}^{\pi} \frac{e^{i \theta}+z}{e^{i \theta}-z} d \sigma(\theta)+i \beta,
$$

where $\sigma(\theta)$ is a monotonic non-increasing function of $\theta$ in the interval $-\pi \leqq \theta \leqq \pi$ and $\beta$ is some real number. There also exists the following relation $\S$ between the function $h(z)$ and the derivative $\sigma^{\prime}(\theta)$ of $\sigma(\theta)$ :

$$
\lim _{z \rightarrow e^{i \theta}} \Re h(z)=\sigma^{\prime}(\theta) \text {, }
$$

for all values of $\theta$ in the interval $-\pi \leqq \theta \leqq \pi$ for which $\sigma(\theta)$ possesses a derivative, the approach $z \rightarrow e^{i \theta}$ being made along any path which is non-tangential to the circle $|z|<1$. Since $g(z)$ is of class $(A)$ in $|z|<1$, the left-hand side of equation (2.2), and therefore $\sigma^{\prime}(\theta)$, is equal to zero almost everywhere. This proves the following theorem:

THEOREM 1. Let $f(z)$ be a function of class $(A)$ in the unit circle $|z|<1$. Then

$$
f(z)=e^{i \beta} B(z) \exp \left[\frac{1}{2 \pi} \int_{-\pi}^{\pi} \frac{e^{i \theta}+z}{e^{i \theta}-z} d \sigma(\theta)\right],
$$

where $B(z)$ is the Blaschke product extended over the zeros of $f(z), \sigma(\theta)$ is a monotonic non-increasing function of $\theta$ in the interval $-\pi \leqq \theta \leqq \pi$ whose derivative $\sigma^{\prime}(\theta)=0$ almost everywhere in $-\pi \leqq \theta \leqq \pi$, and $\beta$ is a real constant. $\|$

It is obvious, conversely, that every function $f(z)$ of the form (2.3) is of class $(A)$ in $|z|<1$.

$\dagger$ Mathematische Zeitschrift, loc. cit.

$\ddagger$ G. Herglotz, Über Potenzreihen mit positivem, reellen Teil im Einheitskreise, Leipziger Berichte, vol. 63 (1911), pp. 501-511; p. 508.

$\S$ Cf. G. C. Evans, The Logarithmic Potential, 1927, pp. 40-43.

\|This is a special case of a result obtained by V. Smirnoff, Sur les valeurs limites des fonctions régulières d l'intérieur d'un cercle, Journal de la Société Physico-Mathématique de Leningrade, vol. 2 (1929), pp. 22-37. 
3. As an immediate consequence of Theorem 1 we prove the following theorem:

THEOREM 2. Let $f(z)$ be a function of class $(A)$, not a constant, in the unit circle $|z|<1$. If $f(z) \neq \alpha(|\alpha|<1)$, in the whole circle $|z|<1$, then there exists at least one radius $\theta=\theta_{0}$ such that

$$
\lim _{r \rightarrow 1} f\left(r e^{i \theta_{0}}\right)=\alpha .
$$

We may assume without loss of generality that $\alpha=0$. For if that is not the case, we prove the theorem for the function

$$
\frac{f(z)-\alpha}{1-\bar{\alpha} f(z)}
$$

which is likewise of class $(A)$ and is different from zero in the whole unit circle. Since $f(z)$ has no zeros in $|z|<1$, formula (2.3) reduces to

$$
f(z)=e^{i \beta} \exp \left[\frac{1}{2 \pi} \int_{-\pi}^{\pi} \frac{e^{i \theta}+z}{e^{i \theta}-z} d \sigma(\theta)\right],
$$

where $\sigma(\theta)$ satisfies the conditions of Theorem 1. The function $\sigma(\theta)$ is not identically a constant, for otherwise $f(z)$ would be constant. Hence, if $\sigma(\theta)$ is continuous, there exists a point $\theta=\theta_{0}$ in the interval $-\pi \leqq \theta \leqq \pi$ at which $\sigma(\theta)$ possesses a derivative equal to $-\infty$. $\dagger$ An easy modification of Evans' proof $\ddagger$ shows that under these conditions the Poisson-Stieltjes integral

$$
u(r, \phi)=\frac{1}{2 \pi} \int_{-\pi}^{\pi} \frac{1-r^{2}}{1+r^{2}-2 r \cos (\theta-\phi)} d \sigma(\theta)
$$

approaches the limit $-\infty$ along the radius $\phi=\theta_{0}$. This proves that $|f(z)|$, which is given by the formula

$$
|f(z)|=e^{u(r, \phi)}, \quad z=r e^{i \phi},
$$

approaches the limit zero along the radius $\phi=\theta_{0}$.

Suppose now that $\sigma(\theta)$ is not continuous in the interval $-\pi \leqq \theta \leqq \pi$. Then, since $\sigma(\theta)$ is non-increasing, it admits the following representation:

$$
\sigma(\theta)=S(\theta)+\Phi(\theta)+\omega(\theta) .
$$

Here all functions $S(\theta), \Phi(\theta), \omega(\theta)$ are non-increasing; $S(\theta)$ is continuous and $S^{\prime}(\theta)=0$ almost everywhere in the interval $-\pi \leqq \theta \leqq \pi, \Phi(\theta)$ is absolutely continuous, and $\omega(\theta)$ is a step function. Since $\sigma^{\prime}(\theta)=0$ almost everywhere in

† Schlesinger and Plessner, Lebesguesche Integrale und Fouriersche Reihen, Berlin and Leipzig, $1926, \$ 43$.

$\ddagger$ The Logarithmic Potential, loc. cit. 
$-\pi \leqq \theta \leqq \pi, \Phi(\theta) \equiv 0$. If $S(\theta)$ is not constant, by the theorem mentioned in the first footnote on page 205, $S^{\prime}(\theta)=-\infty$ at a non-denumerable set of points. Among them there will be at least one point at which $\omega(\theta)$ is continuous. Hence, we may apply to this point the modification of Evans' proof already indicated. There only remains now the case when $S(\theta)$ is constant. In that case $\omega(\theta)$ is certainly not constant. It will have at least one point of discontinuity which, without loss of generality, we may assume to be $\theta=0$. The Poisson-Stieltjes integral on the radius $\theta=0$,

$$
u(r, 0)=\frac{1}{2 \pi} \int_{-\pi}^{\pi} \frac{1-r^{2}}{1+r^{2}-2 r \cos \theta} d \omega(\theta),
$$

will be of the form

$$
J_{0} \frac{1+r}{1-r}+\sum_{k=1}^{\infty} J_{k} \frac{1-r^{2}}{1+r^{2}-2 r \cos \theta_{k}}
$$

where $\theta=\theta_{k}$ are the points of discontinuity of $\omega(\theta)$ at which $\omega(\theta)$ has the negative jumps $J_{k}$, while $J_{0}$ is the jump of $\omega(\theta)$ at the point $\theta=0$. We now choose any positive number $\epsilon$ less than $\left|J_{0}\right|$. To this number there corresponds a positive integer $n$ such that

$$
\sum_{k=n+1}^{\infty}\left|J_{k}\right|<\epsilon .
$$

The sum in (3.2) will now be decomposed in the following manner:

(3.3) $u(r, 0)=J_{0} \frac{1+r}{1-r}+\sum_{k=1}^{n} J_{k} \frac{1-r^{2}}{1+r^{2}-2 r \cos \theta_{k}}+\sum_{k=n+1}^{\infty} J_{k} \frac{1-r^{2}}{1+r^{2}-2 r \cos \theta_{k}}$.

We may disregard the sum

$$
\sum_{k=1}^{n} J_{k} \frac{1-r^{2}}{1+r^{2}-2 r \cos \theta_{k}}
$$

since it tends to 0 as $r$ tends to 1 . The two remaining terms in (3.3) are algebraically less than

$$
-\left(\left|J_{0}\right|-\epsilon\right) \frac{1+r}{1-r}
$$

which tends to $-\infty$ as $r$ tends to 1 .

This completes the proof of Theorem 2.

It is evident that Theorem 2 still holds if the function $f(z)$ is allowed to assume the value $\alpha$ only a finite number of times in the circle $|z|<1$. 
4. By essentially the same method one may prove the following extension of Theorem 2:

THEOREM 3. Let $f(z)$ be a bounded analytic function, not a constant, in the circle $|z|<1$ :

$$
|f(z)|<1 \text {. }
$$

Denoting by $f^{*}\left(e^{i \theta}\right)$ the boundary function of $f(z)$, let

$$
\left|f^{*}\left(e^{i \theta}\right)\right|=1
$$

for almost all values of $\theta$ in the interval $A$ : $\left(0 \leqq \alpha_{1}<\theta<\alpha_{2}<2 \pi\right)$. If the value $\alpha(|\alpha|<1)$ is omitted by $f(z)$, then the set of singularities of $f(z)$ on the arc $A$ is the closed cover of the set of points defined by the solutions of the equation $f^{*}\left(e^{i \theta}\right)$ $=\alpha$.

We may assume, as in the proof of the preceding theorem, that $\alpha=0$. The representation (3.1) is still valid for the function $f(z)$, where $\sigma(\theta)$ is a monotonic non-increasing function in the interval $-\pi \leqq \theta \leqq \pi$, the relation $\sigma^{\prime}(\theta)=0$, however, holding almost everywhere in the open interval $\alpha_{1}<\theta<\alpha_{2}$. If the function $f(z)$ is analytic on the whole arc $A$, then $\left|f^{*}\left(e^{i \theta}\right)\right|=1$ for all points of $A$. Therefore, the equation $f^{*}\left(e^{i \theta}\right)=0$ has no solutions in the interval $\alpha_{1}<\theta$ $<\alpha_{2}$. It is immediately evident that the set of singularities of $f(z)$ on the arc $A$ contains all points of the closed cover of the set defined by the equation $f^{*}\left(e^{i \theta}\right)=0$. In fact, every point $z=e^{i \theta}$ of the $\operatorname{arc} A$ for which $f^{*}\left(e^{i \theta}\right)=0$ is a singular point of $f(z)$. We therefore have to show now that if $P$ is a singular point of $f(z)$ lying on the $\operatorname{arc} A$, then either $f^{*}(P)=0$ or the points defined by the solutions of the equation $f^{*}\left(e^{i \theta}\right)=0$ have $P$ as a limit point. Denote by $\Delta$ an arbitrarily small arc of $|z|=1$ which contains the point $P$ in its interior. The function $\sigma(\theta)$ cannot remain constant on the arc $\Delta$. Indeed, if $\sigma(\theta)$ were constant on $\Delta$, it would follow from equation (3.1) that

$$
f(z)=e^{i \beta} \exp \left[\frac{1}{2 \pi} \int_{C \Delta} \frac{e^{i \theta}+z}{e^{i \theta}-z} d \sigma(\theta)\right]
$$

where $C \Delta$ denotes the arc of the circle $|z|=1$ complementary to the arc $\Delta$. This shows that $f(z)$ is analytic on $\Delta$. Hence, if $\sigma(\theta)$ is continuous on $A$, there must exist a point of the arc $\Delta$ at which $\sigma^{\prime}(\theta)=-\infty$. Since $\Delta$ was taken arbitrarily small, this means that either $\sigma^{\prime}(P)=-\infty$ or the points at which $\sigma^{\prime}(\theta)=-\infty$ have $P$ as a limit point. But now if $\sigma^{\prime}(\theta)=-\infty$, for some $\theta$, then $f^{*}\left(e^{i \theta}\right)=0$ for the same value of $\theta$. If $\sigma(\theta)$ is not continuous on $A$, we can reason in a manner similar to that used in the proof of Theorem 2 . This proves the theorem. 
5. Dr. J. L. Doob kindly pointed out to the author that, as a consequence of Theorem 3, we may prove the following theorem which is a sharper form of Nevanlinna's theorem to be mentioned in $\$ 8$, page 211:

THEOREM 4. Let $w=f(z)$ be a bounded analytic function, not a constant, in the circle $|z|<1$ :

$$
|f(z)|<1 \text {. }
$$

Let $0 \leqq \alpha_{1}<\theta<\alpha_{2}<2 \pi, r=1$, be an arc $A$ of the circumference $|z|=1$ such that

$$
\lim _{r \rightarrow 1} f\left(r e^{i \theta}\right)=f^{*}\left(e^{i \theta}\right), \quad\left|f^{*}\left(e^{i \theta}\right)\right|=1,
$$

for almost all values of $\theta$ in the interval $\alpha_{1}<\theta<\alpha_{2}$. Then if all these limit values $f^{*}\left(e^{i \theta}\right)$ of modulus one are represented by a set of points $E$ on the circumference $|w|=1$, either the set $E$ is the whole circumference $|w|=1$, or $f(z)$ may be continued analytically beyond the arc $A$.

Let $\alpha=e^{i \lambda}$, where $\lambda$ is a real constant, be an arbitrary point of the circumference $|w|=1$ and let the arc $A$ contain in its interior a singular point $P$ of $f(z)$. We wish to prove that there exists a point $e^{i \theta_{0}}$ on the arc $A$ for which $f^{*}\left(e^{i \theta_{0}}\right)=\alpha$.

Consider the function

$$
\phi(z)=e^{(f(z)+\alpha) /(f(z)-\alpha)} .
$$

This function is analytic and bounded in the circle $|z|<1$, and

$$
\lim _{r \rightarrow 1} \phi\left(r e^{i \theta}\right)=\phi^{*}\left(e^{i \theta}\right), \quad\left|\phi^{*}\left(e^{i \theta}\right)\right|=1,
$$

for almost all values of $\theta$ in the interval $\alpha_{1}<\theta<\alpha_{2}$. Furthermore, the point $P$ is a singular point of the function $\phi(z)$. For, since $P$ is a singular point of $f(z)$ according to Theorem 8 , to be proved in $\S 10$, there exists at least one value $c$ $(|c|<1)$ such that the equation $f(z)=c$ has infinitely many solutions $z_{1}$, $z_{2}, \cdots$ interior to the unit circle $|z|<1$ and converging to the point $P$. Hence, it follows from equation (5.1) that

$$
\phi\left(z_{n}\right)=e^{(c+\alpha) /(c-\alpha)}
$$$$
(n=1,2, \cdots) \text {. }
$$

If $P$ were a regular point of $\phi(z)$, the function $\phi(z)$, and hence also $f(z)$, would be constant. Now, according to (5.1) $\phi(z) \neq 0$ in the circle $|z|=1$. Hence, by Theorem 3 there exists at least one radius $\theta=\theta_{0}$ terminating in a point $z=e^{i \theta_{0}}$ of the arc $A$ such that

$$
\lim _{r \rightarrow 1} \phi\left(r e^{i \theta_{0}}\right)=\phi^{*}\left(e^{i \theta_{0}}\right)=0 .
$$

Hence, $f^{*}\left(e^{i \theta_{0}}\right)=\alpha$, as was to be proved. 
6. Theorem 2 may be made to yield the following theorem:

THEOREM 5. Let $f(z)$ be a function of class $(A)$ in the unit circle $|z|<1$. Then, either $f(z)$ is a rational function of the form

$$
f(z)=e^{i \beta_{z}} \prod_{i=1}^{m-k} \frac{z-a_{i}}{1-\bar{a}_{i z}},
$$

where $k$ and $m$ are positive integers, $\beta$ a real number, and $a_{i}$ complex numbers of modulus less than one, giving the most general $(1, m)$ conformal representation of the unit circle into itself; or each value $\alpha(|\alpha|<1)$ belongs to the cluster set $C(P)$ of $f(z)$ in at least one point $P$ of the circumference $|z|=1$.

Suppose some number $a(|a|<1)$ belongs to no cluster set $C(P)$ of $f(z)$. Forming the function

$$
\phi(z)=\frac{f(z)-a}{1-\bar{a} f(z)},
$$

we see that $\phi(z)$ is analytic and bounded in $|z|<1$ :

$$
|\phi(z)|<1
$$

and since $f(z)$ is of class $(A)$, the boundary function $\phi^{*}\left(e^{i \theta}\right)$ satisfies the relation

$$
\left|\phi^{*}\left(e^{i \theta}\right)\right|=1
$$

for almost all values of $\theta$ in the interval $0 \leqq \theta \leqq 2 \pi$. Furthermore, the value 0 belongs to no cluster set $C(P)$ of $\phi(z)$. Hence, there exist at most a finite number of points $z_{1}, z_{2}, \cdots, z_{m}$ of $|z|<1$ at which $\phi(z)$ vanishes. Letting, therefore,

$$
\phi(z)=\prod_{k=1}^{m} \frac{z-z_{k}}{1-\bar{z}_{k} z} \psi(z),
$$

we have by Schwarz's Lemma that $\Psi(z)$ is analytic and bounded in $|z|<1$ satisfying there the inequality

$$
|\psi(z)|<1
$$

According to (6.3) its boundary function $\psi^{*}\left(e^{i \theta}\right)$ is 1 in modulus:

$$
\left|\psi^{*}\left(e^{i \theta}\right)\right|=1
$$

for almost all values of $\theta$ in the interval $0 \leqq \theta \leqq 2 \pi$. Finally, $\psi(z) \neq 0$ in $|z|<1$ and 0 belongs to no cluster set $C(P)$ of $\psi(z)$. Since, as shown by $(6.5), \psi(z)$

† G. Julia, loc. cit., p. 67. 
is of class $(A)$, it follows from Theorem 2, that $\psi(z)$ is identically a constant of modulus one. It is now easily seen with the aid of the relation (6.4) that $f(z)$ admits the representation (6.1).

7. We now consider a certain extension of Schwarz's reflection principle which will be found useful in the sequel. It may be formulated in the following manner:

THEOREM 6. Let $f(z)$ be a bounded analytic function in the unit circle $|z|<1$ :

$$
|f(z)|<1 \text {. }
$$

Let $0 \leqq \alpha_{1}<\theta<\alpha_{2}<2 \pi, r=1$, be an arc $A$ of the periphery of the unit circle such that

$$
\lim _{r \rightarrow 1}\left|f\left(r e^{i \theta}\right)\right|=1
$$

for almost all values of $\theta$ in the interval $\alpha_{1}<\theta<\alpha_{2}$. Then, either $f(z)$ may be continued analytically beyond the arc $A$ or every value $\alpha(|\alpha|<1)$ belongs to at least one of the cluster sets $C(P)$ of $f(z)$ for some point $P$ of $A$.

The theorem need merely be proved for the value $\alpha=0$. Indeed, let us assume that the theorem is true for $\alpha=0$ and let $\beta \neq 0$ be some other value such that $|\beta|<1$. We wish to show that unless $f(z)$ may be continued analytically beyond the arc $A$, the value $\beta$ is contained in at least one of the sets $C(P)$. For suppose that were not the case. Consider the function

$$
\phi(z)=\frac{f(z)-\beta}{1-\bar{\beta} f(z)} .
$$

Then, clearly $|\phi(z)|<1$ and for almost all values of $\theta$ in the interval $\alpha_{1}<\theta<\alpha_{2}$ we have

$$
\lim _{r \rightarrow 1}\left|\phi\left(r e^{i \theta}\right)\right|=1 .
$$

Furthermore, we know that $\phi(z)$ may not be continued analytically beyond the $\operatorname{arc} A$ and the value 0 is contained in none of the sets $C(P)$ formed for the function $\phi(z)$. This, however, contradicts our hypothesis, according to which the theorem was true for the value $\alpha=0$.

In order to prove the theorem for $\alpha=0$, we observe that if the value 0 is contained in none of the sets $C(P)$ formed for the function $f(z)$, there exists a region $R$ lying in the interior of the unit circle and with the $\operatorname{arc} A$ as part of its boundary, in which $1>|f(z)|>\rho$, where $\rho$ is some positive number. The remainder of the proof is analogous to the standard proof of Schwarz's reflection principle. The obvious modifications can be easily supplied by the reader. 
8. As corollary to Theorem 6 we obtain the following result:

COROLLARY. Let $f(z)$ be a bounded analytic function in the unit circle $|z|<1:|f(z)|<1$.

Let $0 \leqq \alpha_{1}<\theta<\alpha_{2}<2 \pi, r=1$, be an arc $A$ of the periphery of the unit circle such that

$$
\lim _{r \rightarrow 1}\left|f\left(r e^{i \theta}\right)\right|=1
$$

for almost all values of $\theta$ in the interval $\alpha_{1}<\theta<\alpha_{2}$. Then, if $P$ is an arbitrary, interior point of $A$, either $f(z)$ is analytic in $P$ or the cluster set $C(P)$ formed for $f(z)$ is the closed unit circle $|\alpha| \leqq 1$.

Suppose $P$ is not a point of analyticity of the function $f(z)$. The corollary follows at once if one applies Theorem 6 to a sequence of arcs $\Delta_{n}$ of the periphery containing the point $P$ whose lengths tend to zero with $1 / n$.

This corollary sharpens Theorem 5 in that in the second case of that theorem every point $P$ of the periphery $|z|=1$ is either a point of analyticity of $f(z)$ or $C(P)$ is the closed unit circle $|\alpha| \leqq 1$.

Finally it may be mentioned in passing that by means of Theorem 6 one may easily prove the following theorem of R. Nevanlinnat:

THEOREM OF R. NEVANLINNA. Let $f(z)=w$ be a bounded analytic function in the unit circle $|z|<1:|f(z)|<1$. Let $\alpha_{1}<\theta<\alpha_{2}, r=1$, be an arc $A$ of the periphery of the unit circle such that

$$
\lim _{r \rightarrow 1} f\left(r e^{i \theta}\right)=f^{*}\left(e^{i \theta}\right), \quad\left|f^{*}\left(e^{i \theta}\right)\right|=1,
$$

for almost all values of $\theta$ in the interval $\alpha_{1}<\theta<\alpha_{2}$. Then, if these limit values $f^{*}\left(e^{i \theta}\right)$ are represented by a set of points $E$ on the periphery of the unit circle $|w|=1$, either the set $E$ is measurable and of measure $2 \pi$, or $f(z)$ may be continued analytically beyond the arc $A$.

9. Again let $w=f(z)$ be a function of class $(A)$ in the circle $|z|<1$. Denote by $z=\phi(w)$ the inverse function of $w=f(z)$. This function is in general infinitely many-valued. In the theorem that follows, we establish a connection between the non-algebraic singularities of the function $\phi(w)$ and the convergence values of the function $f(z)$ in precisely the same manner that Hurwitz and Iversen $\ddagger$ established such a connection for functions meromorphic in the finite plane. Before stating the theorem, we shall give Bieberbach's definition of a singular point:

$\dagger$ R. Nevanlinna, Annales Academiae Scientiarum Fennicae, loc. cit., p. 28.

‡ A. Hurwitz, Sur les points critiques des fonctions inverses, Paris Comptes Rendus, vol. 143 (1906), pp. 877-879, and vol. 144 (1907), pp. 63-65; F. Iversen, Recherches sur les Fonctions Inverses des Fonctions Meromorphes, Thèse, Helsingfors, 1914, p. 13. 
If the members of a chain of regular elements $\mathfrak{B}(w-a)$ of the function $z=\phi(w)$ are obtainable from one another by direct continuation in such a manner that their centers have a single limit point and their radii of convergence tend to zero, then this chain is said to define a singular point. If $w=\alpha$ is the coordinate of the limit point, the singular point is said to lie over the point $w=\alpha$ of the $w$. plane. If the singular point is not algebraic in character, it is said to be nonalgebraic. $\dagger$

The theorem which we shall find useful in the sequel is as follows:

TheоReм 7. Let $w=f(z)$ be a function of class $(A)$ in the unit circle $|z|<1$. If to some number $\alpha(|\alpha|<1)$, there exists a radius $\theta=\theta_{0}$ for which

$$
\lim _{r \rightarrow 1} f\left(r e^{i \theta_{0}}\right)=\alpha,
$$

then the inverse function $z=\phi(w)$ of $w=f(z)$ has a non-algebraic singularity over the point $w=\alpha$. And, conversely, if $\phi(w)$ has a non-algebraic singularity over the point $w=\alpha$, then there exists at least one radius $\theta=\theta_{0}$, for which

$$
\lim _{r \rightarrow 1} f\left(r e^{i \theta_{0}}\right)=\alpha .
$$

Since the proof of this theorem is almost identical with that of Iversen's theorem, we shall omit it here.

10. We now turn to the study of the ranges of values $R(P)$ of our functions. As an immediate consequence to the corollary of Theorem 6, we state the following theorem:

THEOREM 8. Let $w=f(z)$ be a bounded analytic function in the unit circle $|z|<1$ :

$$
|f(z)|<1 \text {. }
$$

Let $0 \leqq \alpha_{1}<\theta<\alpha_{2}<2 \pi, r=1$, be an arc $A$ of the periphery of the unit circle such that

$$
\lim _{r \rightarrow 1}\left|f\left(r e^{i \theta}\right)\right|=1
$$

for almost all values $\theta$ in the interval $\alpha_{1}<\theta<\alpha_{2}$. Then, if $P$ is a singular point of $f(z)$ lying in the interior of the arc $A$, the range of values $R(P)$ is a set of points everywhere dense in the unit circle $|w|<1$.

Consider a sequence of circles $\left\{C_{n}\right\}$ about the point $P$ as center with radii tending to zero. Denote by $V_{n}$ the set of values which $f(z)$ assumes in that part of the circle $C_{n}$, exclusive of its periphery, which lies in the circle

† For further details concerning these matters, cf. L. Bieberbach, Lehrbuch der Funktionentheorie, vol. 1 (1923), pp. 207-217. 
$|w|<1$. Each of the sets $V_{n}$ is open and by the corollary to Theorem 6 is everywhere dense in the circle $|w|<1$. Furthermore, we have

$$
R(P)=\prod_{n=1}^{\infty} V_{n}
$$

Hence, by a well known theorem $\dagger$, the set $R(P)$ is dense in the unit circle $|w|<1$.

11. Under more restrictive hypotheses it is possible to give a sharper statement of Theorem 8 .

THEOREM 9. If $w=f(z)$ is a bounded analytic function in the unit circle $|z|<1$ whose boundary function $f^{*}\left(e^{i \theta}\right)$ satisfies the condition $\left|f^{*}\left(e^{i \theta}\right)\right|=1$ for all values of $\theta$ in the interval $0 \leqq \theta \leqq 2 \pi$ except perhaps in a denumerable set, then either $f(z)$ is of the form (1.1) or it assumes infinitely often all values of the unit circle $|w|<1$ except perhaps for a denumerable set of values.

According to Theorem 2, if $f(z)$ omits (or assumes a finite number of times) a value $w(|w|<1)$, then there exists at least one radius $\theta=\theta_{0}$, such that

$$
\lim _{r \rightarrow 1} f\left(r e^{i \theta_{0}}\right)=w .
$$

Hence, unless the set of values $w$ which is omitted (or assumed only a finite number of times) is denumerable, the function $\left|f^{*}\left(e^{i \theta}\right)\right|<1$ for a non-denumerable set of values in the interval $0 \leqq \theta \leqq 2 \pi$.

The following still sharper theorem was obtained by the author $\ddagger$ in another paper:

Let $w=f(z)$ be a bounded analytic function in the circle $|z|<1:|f(z)|<1$. Let $\left\{n_{k}\right\}$ be an infinite sequence of points interior to the unit circle converging toward $z=1$ in which $f(z)$ vanishes and let $A$ be an arc of the unit circle, $-\alpha<\theta$ $\left\langle\alpha, z=e^{i \theta}\right.$, containing $P:(z=1)$, on which $f(z)$ is continuous except for $P$ and assumes values of modulus one. Then $R(P)$ is the unit circle $|w|<1$ with the exception of at most one point.

As a corollary to this theorem, we may state the following result:

THEOREM 10. Let $w=f(z)$ be of class $(A)$ in the unit circle $|z|<1$. If $f(z)$ omits two or more values of modulus less than one, the set of singularities of $f(z)$ on the circumference $|z|=1$ is perfect.

† Cf. C. Carathéodory, Vorlesungen über reelle Funktionen, Leipzig and Berlin, 1927, p. 63, Theorem 5.

$\ddagger$ These Transactions, loc. cit., p. 17 . 
If $f(z)$ is analytic on the boundary $|z|=1$, then $f(z)$ is necessarily a rational function of the form (1.1). Since, however, the function (1.1) assumes all values of modulus less than one, it follows that $f(z)$ possesses a singularity $P$ on the circumference $|z|=1$. This singularity $P$ cannot be isolated. For if that were the case, there would exist by Theorem 8 a number $c(|c|<1)$, and a sequence of points $z=n_{k}$ interior to the circle $|z|<1$ and converging toward $P$ such that $f\left(n_{k}\right)=c$. If we now form the function $(f(z)-c) /(1-\bar{c} f(z))$ and apply the theorem just quoted, we obtain a contradiction. Since the set of singularities of $f(z)$ is closed and cannot have isolated points, it is perfect.

12. We shall now investigate the set $\Sigma$ of those values which functions $f(z)$ of class $(A)$ assume infinitely often in the unit circle.

$A$ function of class $(A)$ may omit one value. This is evidently true of the function $f(z)=e^{(z+1) /(z-1)}$ which omits the value 0 .

Furthermore, even a Blaschke product $\dagger$, being of class $(A)$, may omit one value. This is readily shown by establishing directly from the Weierstrass product formula the following identity:

$$
\frac{\exp \left[\frac{z+1}{z-1}\right]-e^{-1}}{1-e^{-1} \exp \left[\frac{z+1}{z-1}\right]}=-z \prod_{n=-\infty}^{\infty}\left[\frac{1-\frac{z}{\pi n i /(\pi n i+1)}}{1+\frac{\pi n i}{-\pi n i+1} z}\left|\frac{\pi n i}{\pi n i+1}\right|\right],
$$

the prime on the product sign indicating that the factor for $n=0$ is omitted. The function on the left evidently omits the value $-e^{-1}$. The function on the right is a Blaschke product of the form (1.2), where

$$
a_{n}=\frac{\pi n i}{\pi n i+1} .
$$

Thus the Blaschke product in (12.1) omits the value $-e^{-1}$. Since $w=e^{(z+1) /(z-1)}$ is the inverse function of that function which maps the punctured circle $0<|w|<1$ on the unit circle $|z|<1$, we find that the inverse function of this mapping function may be represented as a linear function of the Blaschke product B(z) in (12.1):

$$
w=\frac{B(z)+e^{-1}}{1+e^{-1} B(z)} .
$$

13. This last result will now be extended in the following manner:

† For Blaschke products cf. $\S 1$ of this paper. 
THEOREM 11. Let $f(z)$ be a function of class $(A)$ in the unit circle $|z|<1$. Let the value $\alpha(|\alpha|<1)$ which is assumed infinitely often by $f(z)$ in the circle $|z|<1$ be not a convergence value of $f(z)$. That is, for no radius $\theta=\theta_{0}$ does the equation

$$
\lim _{r \rightarrow 1} f\left(r e^{i \theta_{0}}\right)=\alpha
$$

hold. Then $f(z)$ is equal to a linear function of a Blaschke product:

$$
f(z)=\frac{e^{i \delta} B(z)+\alpha}{1+\bar{\alpha} e^{i \delta} B(z)} .
$$

For consider the function

$$
f_{1}(z)=\frac{f(z)-\alpha}{1-\bar{\alpha} f(z)} .
$$

By hypothesis $f_{1}(z)$ has infinitely many zeros in points $a_{1}, a_{2}, \cdots, a_{i}, \cdots$. By Theorem $1 f_{1}(z)$ can be represented in the following manner:

$$
f_{1}(z)=e^{i \delta} B(z) \exp \left[\frac{1}{2 \pi} \int_{-\pi}^{\pi} \frac{e^{i \theta}+z}{e^{i \theta}-z} d \sigma(\theta)\right],
$$

where $\delta$ is a real constant, $B(z)$ the Blaschke product extended over the zeros of $f_{1}(z)$, and $\sigma(\theta)$ a monotonic non-increasing function of $\theta$ in the interval $-\pi \leqq \theta \leqq \pi$ for which $\sigma^{\prime}(\theta)=0$ in almost all points of $-\pi \leqq \theta \leqq \pi$. Now if $\sigma(\theta)$ is not constant in the whole interval $-\pi \leqq \theta \leqq \pi$, by the reasoning of $\S 3$ there exists a value $\theta_{0}$ of $\theta$ such that $f_{1}^{*}\left(e^{i \theta_{0}}\right)=0$ or $f^{*}\left(e^{i \theta_{0}}\right)=\alpha$. This contradicts the non-existence of the relation (13.1). Hence, $\sigma(\theta)$ is a constant and formula (13.3) reduces to

$$
f_{1}(z)=e^{i \delta} B(z) \text {. }
$$

14. Theorem 11 leads immediately to the following result:

THEOREM 12. Let $f(z)$ be a function of class $(A)$ in the circle $|z|<1$. Then either $f(z)$ is a linear function of a Blaschke product, or to every number $\alpha$ $(|\alpha|<1)$ there exists at least one radius $\theta=\theta_{0}$ 'on which $f(z)$ tends to the value $\alpha$ :

$$
\lim _{r \rightarrow 1} f\left(r e^{i \theta_{0}}\right)=\alpha .
$$

By Theorem 7 in the second case, the Riemann surface of the inverse function $z=\phi(w)$ of $w=f(z)$ has non-algebraic singularities over every point of the unit circle $|w|<1$.

If $f(z)$ is not a linear function of a Blaschke product, then according to Theorem 11 , every value $\alpha(|\alpha|<1)$ which is assumed infinitely often by 
$f(z)$ is also a convergence value of $f(z)$. But we know from Theorem 2 that every value $\alpha(|\alpha|<1)$ which is omitted (or assumed only a finite number of times) is a convergence value of $f(z)$. Hence every value $\alpha(|\alpha|<1)$ is a convergence value of $f(z)$. The author has been unable to determine whether or not functions of the second kind may actually exist.

15. We may now return to the study of the sets $\Sigma$ of values which functions of class $(A)$ assume infinitely often in the unit circle $|z|<1$. We have seen in $\$ 12$ that there are functions of class $(A)$ which omit one value of modulus less than one. It is an easy matter to obtain functions of class $(A)$ which omit a non-denumerable infinity of values of modulus less than one.

In order to construct functions of this kind, consider a set $S$ of interior points of the circle $|w|<1$. Let us assume that the set $S$ is closed relatively to the circle and that it has the following property: to every $\epsilon>0$ there corresponds a sequence of circles $\left\{C_{n}\right\}$ which cover the set $S$ and whose radii $\delta_{n}$ satisfy the inequality

$$
\sum_{n=1}^{\infty} \frac{1}{\log ^{+}\left(1 / \delta_{n}\right)}<\epsilon .
$$

A set with this property is said to be of logarithmic measure zero. If the points of the set $S$ are removed from the interior of the circle $|w|<1$, there remains an open set of points. If this open set is connected, we denote the resulting region by $R_{S}$. If this open set is not connected (as is a priori conceivable), we denote by $R_{S}$ an arbitrary one of the regions into which the open set is decomposed by $S$. It will be shown a little later that $R_{S}$ is always a dense set in the circle $|w|<1$, thus proving that the open set in question is always connected.

By the fundamental theorem of conformal mapping there exists a function $z=\phi(w)$ which maps the region $R_{S}$ conformally on the unit circle $|z|<1$ of the $z$-plane. This function $\phi(w)$ is infinitely multiple-valued in the region $R_{S}$ if $R_{S}$ is multiply connected. The Riemann surface $\Re$ of $\phi(w)$ is the universal covering surface $\dagger$ of the region $R_{S}$. Such a covering surface is unramified relatively to $R_{S}$ and consequently can have non-algebraic singularities only over the points of the set $S$. Let us now consider the inverse function $w=f(z)$ of $z=\phi(w)$. This function $f(z)$ is always single-valued, analytic, and bounded in the circle $|z|<1:|f(z)|<1$. The boundary function $f^{*}\left(e^{i \theta}\right)$ is defined by Fatou's theorem in almost all points of the circumference $r=1$, $0 \leqq \theta \leqq 2 \pi$. Denote by $E$ the set of all those points of the circumference at which $f^{*}\left(e^{i \theta}\right)$ assumes a value belonging to the set $S$. Since $S$ is of logarithmic

$\dagger$ For a detailed account of the subject of conformal mapping of multiply connected regions, cf. L. Bieberbach, Lehrbuch der Funktionentheorie, vol. 2, 2d edition, 1931, chapters I and IV, as well as H. Weyl, Die Idee der Riemannschen Fläche, 1913, chapter I, especially $\$ 9$. 
measure zero, it readily follows from a theorem of $\mathrm{R}$. Nevanlinna $\dagger$ that the set $E$ is linearly measurable and of measure zero. Furthermore, it may be shown that the equation $f^{*}\left(e^{i \theta}\right)=\alpha$, where $\alpha$ is any complex number of modulus less than one, has a solution if, and only if, the point $w=\alpha$ is a point of the set $S$. Hence, $\left|f^{*}\left(e^{i \theta}\right)\right|<1$ for a set of values of $\theta$ which is of measure.zero. Thus, for almost all values of $\theta$ we have $\left|f^{*}\left(e^{i \theta}\right)\right|=1$. This shows that the function $f(z)$ is of class $(\dot{A})$. On the other hand, it is immediately apparent from the definition of $f(z)$ that $f(z)$ omits in the circle $|z|<1$ all values corresponding to points of the set $S$. This proves our initial assertion.

Incidentally, we notice that, according to Theorem $5, f(z)$, being of class $(A)$ and possessing singularities on the boundary $|z|=1$, assumes a set of values everywhere dense in the circle $|z|<1$. This proves that the region $R_{S}$ is everywhere dense in the circle $|w|<1$. Hence, if the set $S$ is removed from the interior of the circle $|w|<1$, there remains a single connected region $R_{S}$.

With the aid of Theorem 11 we now prove the following theorem:

THEOREM 13. Let $S$ be a set of interior points of the circle $|w|<1$ which is closed relatively to the circle and of logarithmic measure zero. Denote by $R_{S}$ the region which is obtained by removing the points af the set $S$ from the interior of the circle $|w|<1$. Let $z=\phi(w)$ be an arbitrary function which maps $R_{S}$ conformally on the circle $|z|<1$. Then the inverse function $w=f(z)$ of $z=\phi(w)$ may be represented as a linear function of a Blaschke product:

$$
f(z)=\frac{e^{i \delta} B(z)+\beta}{1+\bar{\beta} e^{i \delta} B(z)}, \delta \text { real, } \quad|\beta|<1 .
$$

From the discussion which has preceded the statement of this theorem, it is evident that $f(z)$ is of class $(A)$ in the circle $|z|<1$ and assumes infinitely often in this circle every value of modulus less than one which corresponds to an interior point of the region $R_{S}$. Furthermore, no such value $\alpha$ can be a convergence value of $f(z)$, for the Riemann surface $\Re_{S}$ is unramified relatively to $R_{S}$. Consequently, according to Theorem 11, whose hypotheses are satisfied by the function $f(z)$, the inverse function $f(z)$ of the mapping function $\phi(w)$ may be represented as a linear function of a Blaschke product $B(z)$ of the form (15.1). Since $f(z)$ clearly omits all values of $S$, this theorem also shows that to any set $S$ of logarithmic measure zero and closed relatively to the circle $|w|<1$ there corresponds a Blaschke product, or a linear function of a Blaschke product, which assumes all values of modulus less than one infinitely often in the unit circle $|z|<1$ save those belonging to the set $S$ which it omits.

$†$ R. Nevanlinna, Über die Randwerte von analytischen Funktionen, Commentarii Mathematici Helvetici, vol. 2 (1930), pp. 237-244. 
16. Theorem 13 is of some interest in the light of a recent remarkable investigation of Besicovitch. $\dagger$ Among other things Besicovitch generalizes Weierstrass's theorem on the behavior of single-valued analytic functions in the neighborhood of an isolated essential singularity to the case of a nonisolated one. His theorem is as follows:

THEOREM OF BESICOVITCH. If the set $E$ of essential singularities of a singlevalued analytic function $f(z)$ is of linear measure zero, then the set of values of $f(z)$ in the neighborhood of each of the points of $E$ is everywhere dense on the complex plane.

It is natural to inquire whether or not it is possible to extend Picard's theorem in an analogous manner. Theorem 13 gives us the means of answering this question negatively, thus showing that the hypothesis of an isolated essential singularity, while not necessary for Weierstrass's theorem, cannot be dropped in Picard's theorem. In fact, we shall prove the following assertion:

There exist single-valued functions $f(z)$ analytic in the whole z-plane except for a set $E$ of essential singularities of linear measure zero and omitting a nondenumerable set $\Sigma$ of values of logarithmic measure zero.

Let us consider an arbitrary, closed, non-denumerable set of points $S$ of logarithmic measure zero containing the origin $w=0$ and lying in the interior of some circle $|w|<\rho<1$. On removing the points of the set $S$ from the circle $|w|<1$, we obtain a region $R_{S}$. Let $w=f(z)$ be the inverse function of that function $z=\phi(w)$ which maps $R_{S}$ conformally on the circle $|z|<1$, carrying three preassigned points of the circumference $|w|=1$ into three preassigned points of the circumference $|z|=1$. According to the result of $\S 15$, the boundary function $f^{*}\left(e^{i \theta}\right)$ has the property that

$$
\left|f^{*}\left(e^{i \theta}\right)\right|=1
$$

for almost all values $\theta$ in the interval $0 \leqq \theta \leqq 2 \pi$.

We shall prove now that every point $z=e^{i \theta_{0}}$ for which $\left|f^{*}\left(e^{i \theta_{0}}\right)\right|=1$ is a point of analyticity of $f(z)$. To this purpose, consider the radius $\theta=\theta_{0}$ and the image $L$ of this radius. by $w=f(z)$ on the universal covering surface $\Re_{S}$ of the region $R_{S}$. It is clear that the projection of the curve $L$ on the w-plane tends to a point $w=e^{i \mu}$ of modulus one. We shall now show that the curve $L$ from a certain point on lies wholly on one sheet of the Riemann surface $\Re_{S}$. If this were not the case, then $L$ would have to wind infinitely many times around branch points. Since, however, $\Re_{S}$ has no branch points in the ring $\rho<|w|$ $<1$, the curve $L$ either would have to wind infinitely often in the ring $\rho<|w|$

$\dagger$ A. S. Besicovitch, On sufficient conditions for a function to be analytic, Proceedings of the London Mathematical Society, (2), vol. 32 (1931), pp. 1-9. 
$<1$ or would have to penetrate the circle $|w|<\rho$ infinitely many times. In either case, its projection would not tend to a single limit point. Hence, the curve $L$ from a certain point on lies on a single sheet of $\Re_{S}$ and terminates in a definite boundary point $P$ of $\Re_{S}$ which lies over the point $w=e^{i \mu}$. Now, the function $z=\phi(w)$ is clearly analytic in the point $P$ and $\phi^{\prime}(P) \neq 0$, since it is an interior point of the free analytic curve $|w|=1$, that is, of a curve whose points are not limit points of boundary points not belonging to the curve. Hence, $f(z)$ is analytic in the point $z=e^{i \theta_{0}}$. Consequently, by virtue of (16.1) $f(z)$ is analytic and of modulus one in almost all points of the circumference $|z|=1$ which form an open, everywhere dense set.

By Schwarz's reflection principle $f(z)$ may be continued analytically across points of this open set on the circumference $|z|=1$ in accordance with the functional relation

$$
f(1 / \bar{z})=(1 / \overline{f(z)}
$$

Since the set $S$ was so chosen as to contain the origin $w=0$ this defines a function $f(z)$ analytic in the whole plane except for a perfect nowhere dense set of linear measure zero of essential singularities on the circumference $|z|=1$. Furthermore, if we denote by $S^{\prime}$ the image set of $S$ by an inversion in the unit circle, it is evident that the function $f(z)$ omits all values belonging to the set $\Sigma=S+S^{\prime}$.

17. It is natural to inquire whether Theorem 13 still holds when the set $S$ of logarithmic measure zero is replaced by a set of linear measure zero. The answer hinges on whether or not the inverse of the mapping function is of class $(A)$. In the sequel we shall prove that in general the question is to be answered in the negative. The sets $S$ with which we shall deal can be characterized as follows:

(1) $S$ is closed.

(2) If the points of $S$ are removed from the plane, the remaining set of points is connected.

(3) There exists a function $s(\rho)$ which is positive, continuous, and monotonically increasing for $\rho>0$ such that the integral

$$
\int_{0}^{k} \frac{s(\rho)}{\rho} d \rho
$$

is finite for some positive value of $k$, and there exists a positive number $\epsilon$ such that for every sequence of circles $\left\{C_{\nu}\right\}$ with the radii $\rho_{\nu}$ which cover $S$ the inequality

$$
\sum_{\nu=1}^{\infty} s\left(\rho_{\nu}\right)>\epsilon
$$


is satisfied. All sets $S$ satisfying conditions 1,2 , and 3 will be said to possess the property $(K)$. Given a set $S$ and a function satisfying condition 3, denote by $C(\lambda)$ the greatest lower bound of sums $\sum_{v=1}^{\infty} s\left(\rho_{v}\right)$ corresponding to a sequence of circles $\left\{C_{\nu}\right\}$ of radii $\rho_{\nu}$ which cover $S$ and such that $\rho_{\nu} \leqq \lambda$. The function $C(\lambda)$ is non-increasing. Hence, $\lim _{\lambda \rightarrow 0} C(\lambda)=m_{s} S$ exists and will be called the $s$-measure of the set $S$. Thus, if $s(\rho)=\rho^{\alpha}(\alpha>0)$, condition 3 states that $S$ is of positive $\alpha$-dimensional measure (in particular, $\alpha=1$ gives linear measure and $\alpha=2$ superficial measure). The $s$-measure of a set, however, may also be defined by means of the function

$$
s(\rho)=\frac{1}{\left(\log +\frac{1}{\rho}\right)^{1+\eta}}
$$

or more generally by means of the function

$$
s(\rho)=\frac{1}{\log ^{+} \frac{1}{\rho} \log _{2}+\frac{1}{\rho} \cdots\left(\log _{m}+\frac{1}{\rho}\right)^{1+\eta}} \quad(\eta>0) .
$$

It is evident that a set $S$ with the property $(K)$ may be of linear measure zero.

In view of a later application which is to be made of the following result, we state it in the form of a lemma:

LEMMA. Let $S$ be a set of points possessing the property $(K)$ and lying in the interior of a circle $|w|<\lambda<1$. If the set $S$ is removed from the interior of the circle $|w|<1$, there remains an open connected region $R_{S}$. Denote by $z=\phi(w) a$ function which maps $R_{S}$ conformally on the unit circle $|z|<1$. Then the inverse function $w=f(z)$ of $z=\phi(w)$ is not of class $(A)$.

The function $w=f(z)$ is bounded in the unit circle $|z|<1:|f(z)|<1$. Consequently, by Fatou's theorem,

$$
\lim _{r \rightarrow 1} f\left(r e^{i \theta}\right)=f^{*}\left(e^{i \theta}\right) \quad\left(z=r e^{i \theta}\right)
$$

exists for almost all values of $\theta$ in the interval $0 \leqq \theta \leqq 2 \pi$. It is to be shown that $\dagger$

We shall assume that

$$
m E\left(\left|f^{*}\left(e^{i \theta}\right)\right|<1\right)>0 .
$$

$$
m E\left(\left|f^{*}\left(e^{i \theta}\right)\right|<1\right)=0,
$$

and derive a contradiction.

$\dagger$ The notation $m E\left(\left|f^{*}\left(e^{i \theta}\right)\right|<1\right)$ is used here to denote the linear measure of that set of points of the circumference $|z|=1$ for which $\left|f^{*}\left(e^{i \theta}\right)\right|<1$. 
We may remove the set $S$ and the point at infinity, $w=\infty$, from the whole w-plane, and thus obtain a new region $\Delta$ which we shall map conformally on the unit circle $|t|<1$ by means of a function $t=\Phi(w)$. Consider the inverse function $w=F(t)$ which is single-valued and analytic in the circle $|t|<1$. R. Nevanlinna proved that $F(t)$ may be represented as the quotient of two functions each of which is analytic and bounded in the circle $|t|<1$. $\dagger$ Hence, as may be easily seen from a theorem of F. and M. Rieszf,

$$
\lim _{\rho \rightarrow 1} F\left(\rho e^{i \tau}\right)=F^{*}\left(e^{i \tau}\right)
$$$$
\left(t=\rho e^{i \tau}\right)
$$

exists for almost all values of $\tau$ in the interval $0 \leqq \tau \leqq 2 \pi$. Nevanlinna further shows that each finite convergence value (17.2), when represented as a point in the w-plane, is a point of the set $S$. Hence

$$
m E\left(\left|F^{*}\left(e^{i \tau}\right)\right|<\lambda\right)=2 \pi,
$$

where by hypothesis $\lambda<1$.

Consider now the set $G$ of all those points of the circle $|t|<1$ in which $|F(t)|<1$. This set of points is evidently open and, as we shall show now, connected. If the set $G$ is not connected, it may be decomposed into two or more connected open subsets $G_{1}, G_{2}, \cdots$. Let $G_{1}$ and $G_{2}$ be any two of these subsets and let $t_{1}$ be a point of $G_{1}$ and $t_{2}$ a point of $G_{2}$. Let $l$ be any continuous path in the circle $|t|<1$ connecting $t_{1}$ and $t_{2}$. Then, there exists at least one point $t_{0}$ on $l$ for which $\left|F\left(t_{0}\right)\right| \geqq 1$. But now $F(t)$ maps the unit circle $|t|<1$ in a one-to-one manner and conformally on the universal covering surface $\Re_{\Delta}$ of the region $\Delta$. Consequently, the curve $l$ is mapped on a curve $L$ lying on the surface $\Re_{\Delta}$ joining two points $w_{1}$ and $w_{2}$ which lie over two interior points of the circle $|w|<1$ and passing through at least one point $w_{0}$ which lies over some point of the closed region $|w| \geqq 1$. We know, however, from the definition of the region $\Delta$ that the surface $\Re_{\Delta}$ is unramified over the region $\lambda<|w|<\infty$. Hence, we may deform the curve $L$ continuously into a curve $L^{\prime}$ which joins the points $w_{1}$ and $w_{2}$ and lies wholly over the interior of the circle $|w|<1$. Hence, the curve $l$ may be deformed continuously into a curve $l^{\prime}$ which joins the two points $t_{1}$ and $t_{2}$ and on which $|F(t)|<1$. Hence, the two sets $G_{1}$ and $G_{2}$ could not have been distinct. This proves that $G$ is a connected open set.

The region $G$ cannot be wholly contained in any circle $|t|<r<1$. Furthermore, as follows easily from the maximum principle of analytic func-

\footnotetext{
$\dagger$ R. Nevanlinna, Commentarii Mathematici Helvetici, loc. cit., pp. 250-252.

$\ddagger$ F. and M. Riesz, Über die Randwerte einer analytischen Funktion, Compte Rendu du Quatrième Congrès des Mathématiciens Scandinaves (1920), pp. 28-30.
} 
tions, the region $G$ is simply connected. Consider now that connected part $\Re_{\Delta}{ }^{\prime}$ of the surface $\Re_{\Delta}$ into which the region $G$ is mapped by the function $w=F(t)$. From the definition of $G$ it follows that $\Re_{\Delta}^{\prime}$ is that part of the surface $\Re_{\Delta}$ which lies over the region $R_{S}$. Furthermore, from the simple connectivity of $G$ follows the simple connectivity of $\Re_{\Delta}{ }^{\prime}$. Hence, $\Re_{\Delta}{ }^{\prime}$ is a simply connected, unramified, and unbounded covering surface of the region $R_{S}$. It follows from this at once that $\Re_{\Delta}{ }^{\prime}$ is the universal covering surface $\Re_{R_{s}}$ of $R_{S}$. To sum up the preceding, we have obtained a simply connected subregion $G$ of the circle $|t|<1$ which is mapped in a one-to-one manner and conformally by $w=F(t)$ on the universal covering surface $\Re_{R_{s}}$ of the region $R_{S}$.

18. Denote by $w=f(z)$ the inverse function of some function $z=\phi(w)$ which maps the region $R_{S}$ conformally on the circle $|z|<1$. The function $w=f(z)$ maps, therefore, the circle $|z|<1$ in a one-to-one manner and conformally on the universal covering surface $\Re_{R_{s}}$ of the region $R_{S}$. Consequently, by virtue of the result of $\S 17$ the function $t=\Phi(f(z))$ establishes a one-to-one conformal map between the circle $|z|<1$ and the subregion $G$ of $|t|<1$.

Before completing the proof of the lemma, it is necessary to make some additional remarks about the region $G$. If the point $t=e^{i \tau_{0}}$ is such that

$$
\lim _{\rho \rightarrow 1} F\left(\rho e^{i \tau_{0}}\right)
$$

exists, then by virtue of the fact that $F(t)$ can be represented as the quotient of two bounded functions and by Lindelöf's theorem it follows that the limit (18.1) exists uniformly in every angle smaller than $180^{\circ}$ whose vertex lies in the point $e^{i r_{0}}$ and whose bisector falls along the radius joining the origin $t=0$ with the point $e^{i \tau_{0}}$. From (17.3) it follows by applying Egoroff's well known theorem that there exists a perfect set $\Omega$ of positive measure on the circumference $|t|=1$ and a positive number $r_{0}<1$ such that if $e^{i r}$ is an arbitrary point of $\Omega$ and $A\left(e^{i r}\right)$ an angle of $60^{\circ}$ whose vertex lies in the point $e^{i r}$ and whose bisector falls on the radius joining the origin $t=0$ with the point $t=e^{i \tau}$, then $|F(t)|<1$ for every point $t$ of the angle $A\left(e^{i \tau}\right)$ whose distance $|t|$ from the origin is greater than $r_{0}$. That part of the angle $A\left(e^{i \tau}\right)$ whose points lie at a distance not less than $r_{0}$ from the origin will be denoted by $B\left(e^{i \tau}\right)$. Since $G$ was defined as the totality of those points of the circle $|t|<1$ for which $|F(t)|<1$, it follows that the region $B\left(e^{i \tau}\right)$ lies wholly within $G$ for every point $e^{i \tau}$ belonging to the set $\Omega$.

Now, the set $C \Omega$ complementary to $\Omega$ is open and consists of denumerably many open $\operatorname{arcs} a_{n} b_{n}$ of the periphery $|t|=1$, no two arcs having any points in common. Through each end point $a_{n}$ and $b_{n}$ draw a line forming an angle of $30^{\circ}$ with the corresponding radius. These two lines intersect in a 
point $c_{n}$. We thus obtain denumerably many circular sectors $a_{n} b_{n} c_{n}$. Consider now a point $t(|t|<1)$, whose distance $|t|$ from the origin $t=0$ is greater than $r_{0}$ and which lies outside of all the sectors $a_{n} b_{n} c_{n}$. Corresponding to this point there exists a point $e^{i r}$ of the set $\Omega$ such that the region $B\left(e^{i \tau}\right)$ contains $t$ in its interior or on its boundary. In either case $t$ is an interior point of $G$. There exist at most a finite number of sectors, which we number $a_{1} b_{1} c_{1}$, $\cdots, a_{M} b_{M} c_{M}$, falling in part within the circle $|t| \leqq r_{0}$. If $n \leqq M$, denote by $a_{n}{ }^{\prime}$ the intersection of $\overline{a_{n} c_{n}}$ with $|t|=r_{0}$ and by $b_{n}{ }^{\prime}$ the intersection of $\overline{b_{n} c_{n}}$ with $|t|=r_{0}$. Let us assume that the sectors $a_{1} b_{1} c_{1}, \cdots, a_{M} b_{M} c_{M}$ have been so numbered that the points $a_{1}, b_{1}, a_{2}, b_{2}, \cdots, a_{M}, b_{M}$ describe the circumference $|t|=1$ in counter-clockwise sense. Then, at least one of the $\operatorname{arcs} b_{1} a_{2}$, $b_{2} a_{3}, \cdots, b_{M-1} a_{M}, b_{M} a_{1}$ contains a subset of positive measure of the set $\Omega$. Denote any such arc by $b_{n} a_{n+1}$. Consider now the contour $b_{n} b_{n}{ }^{\prime} a_{n+1}^{\prime} a_{n+1}$ as shown in the figure. If we remove all the circular sectors which fall within the region bounded by the contour $b_{n} b_{n}{ }^{\prime} a_{n+1}^{\prime} a_{n+1}$, we obtain a new region $G^{\prime}$ which is a subregion of $G$. The boundary of the region $G^{\prime}$ is by construction a closed rectifiable Jordan curve and it has a set of points of positive measure in common with the circumference $|t|=1$, namely that subset of $\Omega$ which lies on the $\operatorname{arc} b_{n} a_{n+1}$.

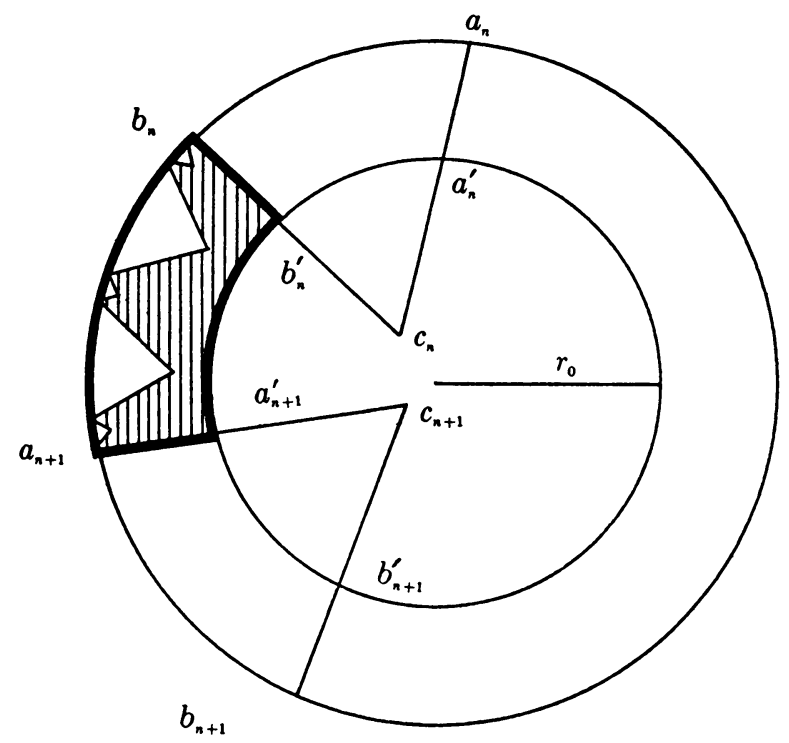

19. We have assumed that (17.1) holds; that is, that the function $f(z)$ is of class $(A)$. Consider some point $P$ of the subset of $\Omega$ which lies on the arc $b_{n} a_{n+1}$. Let $\gamma$ be an arc which lies in the region $G^{\prime}$ except for its end point $P$. The function $w=F(t)$ maps it on an arc $\gamma^{\prime}$ lying on the Riemann surface 
$\Re_{R_{s}}$ and terminating in some point which lies over a point of the set $S$. The function $z=\phi(w)$ maps $\gamma^{\prime}$ on an arc $\gamma^{\prime \prime}$ which terminates in some point $P^{\prime \prime}$ of $|z|=1$ belonging to the set $E\left(\left|f^{*}\left(e^{i \theta}\right)\right|<1\right)$ which by (17.1) is of measure zero.

Consider now a function $\nu(z)$ defined and bounded in the unit circle $|z|<1:|\nu(z)|<1$, and such that it does not tend to any limit along all paths terminating in points of the set $E\left(\left|f^{*}\left(e^{i \theta}\right)\right|<1\right)$. That such functions exist is known from a result of Lusin and Priwaloff. $\dagger$ We have seen in the beginning of $\S 18$ that the function $t=\Phi(f(z))$ maps the circle $|z|=1$ in a one-to-one manner on the region $G$. Denote by $z=g(t)$ the inverse function and consider the new function $\mu(t)=\nu(g(t))$. It follows from the last paragraph that $\mu(t)$ approaches no limit along any path of the region $G$ (and in particular of the region $G^{\prime}$ ) terminating in points of that subset of $\Omega$ which lies on the $\operatorname{arc} b_{n} a_{n+1}$. We thus obtain a bounded analytic function $\mu(t)$ in a region $G^{\prime}$ bounded by a single rectifiable Jordan curve and a set of boundary points of $G^{\prime}$ of positive linear measure such that $\mu(t)$ tends to no limit along any path in $G^{\prime}$ terminating in a point of the set. This, however, contradicts a known theorem. $\ddagger$ Thus the assumption (17.1) leads to a contradiction. Consequently, $m E\left(\left|f^{*}\left(e^{i \theta}\right)\right|<1\right)>0$, and $f(z)$ is not of class $(A)$.

20. By means of the lemma just established we prove

THEOREM 14. Let $f(z)$ be a function which is analytic and bounded in the unit circle $|z|<1:|f(z)|<1$. Let $A: \alpha_{1}<\theta<\alpha_{2}, r=1$, be an arc of the circumference $|z|=1$ such that

$$
\lim _{r \rightarrow 1} f\left(r e^{i \theta}\right)=f^{*}\left(e^{i \theta}\right), \quad\left|f^{*}\left(e^{i \theta}\right)\right|=1,
$$

for almost all values of $\theta$ in the interval $\alpha_{1}<\theta<\alpha_{2}$. Then either $f(z)$ is analytic on the arc $A$ or $f(z)$ assumes in the circle $|z|<1$ every value $\alpha(|\alpha|<1)$ save perhaps for a set $S$ of such values possessing the following property: For every function $s(\rho)$ positive, continuous, and monotonically increasing in $\rho>0$ such that the integral

$$
\int_{0}^{k} \frac{s(\rho)}{\rho} d \rho
$$

is finite for some $k$, and for any positive $\epsilon$ there exists a sequence of circles $\left\{C_{\nu}\right\}$ with radii $\rho_{\nu}$ covering the set and such that $\sum_{\nu=1}^{\infty} s\left(\rho_{\nu}\right)<\epsilon$.

$\nmid$ N. Lusin and J. Priwaloff, Sur l'unicité et la multiplicité des fonctions analytiques, Annales Scientifiques de l'Ecole Normale Supérieure, (3), vol. 42 (1925), pp. 157-159.

$\ddagger$ V. V. Golubev, Single-valued analytic functions with perfect singular sets (in Russian), Moscow, 1916, p. 44. Cf. also F. and M. Riesz, loc. cit., p. 40. 
Let the function $f(z)$ be not analytic on the $\operatorname{arc} A$. If the set $S$ of values in the $w$-plane which $w=f(z)$ omits does not satisfy the condition of Theorem 14 , then the set possesses the property $(K)$ defined in $\$ 17$. Thus, for some function $s(\rho)$ the $s$-measure of the set $S$ is positive. Consider a monotonically increasing sequence of positive numbers $\left\{r_{n}\right\}$ which tend to 1 in the limit. Denote by $S_{1}$ that part of $S$ which lies in the circle $|z|<r_{1}$, and in general by $S_{n}$ that part of $S$ which lies in the ring $r_{n-1} \leqq|z|<r_{n}$. Then $S=\sum_{\nu=1}^{\infty} S_{\nu}$. Furthermore, it follows immediately from the definition of $s$-measure that

$$
m_{s} S \leqq m_{s} S_{1}+m_{s} S_{2}+\cdots+m_{s} S_{m}+\cdots .
$$

Hence, if the $s$-measure of $S$ is positive, then the $s$-measure of at least one set $S_{n}$ is likewise positive. Consider any such set $S_{n}$. It possesses the property $(K)$ and lies in the interior of some circle $|w|<\lambda<1$. If the set $S_{n}$ is removed from the interior of the circle $|w|<1$, there remains an open connected region $R_{S_{n}}$. Denote by $t=\phi(w)$ some function which maps the region $R_{S_{n}}$ on the unit circle $|t|<1$. By the lemma stated in $\$ 17$ the inverse function $w=\psi(t)$ of $t=\phi(w)$ is not of class $(A)$. That is to say, the relations

$$
\lim _{\sigma \rightarrow 1} \psi\left(\sigma e^{i \tau}\right)=\psi^{*}\left(e^{i \tau}\right), \quad\left|\psi^{*}\left(e^{i \tau}\right)\right|<\lambda<1, \quad t=\sigma e^{i \tau},
$$

hold for a set of positive measure of values of $\tau$ in the interval $0 \leqq \tau \leqq 2 \pi$.

Consider now the function $t=\phi[f(z)]$. Since $f(z)$ does not assume any value of the set $S_{n}$ and since $\phi(w)$ has the points of $S_{n}$ as its only singularities, it follows that the function $\phi[f(z)]$ may be continued analytically along every path lying in the interior of the unit circle $|z|<1$. By the monodromy theorem, therefore, the function $\phi[f(z)]$ is analytic and single-valued in the circle $|z|<1$ as soon as some definite branch of $\phi(w)$ has been selected. It is furthermore immediately evident that

$$
|\phi[f(z)]|<1
$$

in the circle $|z|<1$. Next, consider any radius $\theta=\theta_{0}$ terminating in a point of the $\operatorname{arc} A$ on which $f(z)$ tends to a limit and $|f(z)|$ tends to the value 1 . Such a radius $\theta=\theta_{0}$ is mapped by $w=f(z)$ on a continuous curve $p_{0}$ in the circle $|w|<1$ terminating in a point $P_{0}$ of the periphery $|w|=1$. But now the function $t=\phi(w)$ maps the curve $p_{0}$ on a curve $\pi_{0}$ in the circle $|t|<1$ terminating in a point $\Pi_{0}$ of the periphery $|t|=1$. Since by hypothesis the relations (20.1) hold for almost all points of the arc $A$, this proves that the function $\phi[f(z)]$ likewise satisfies the conditions

$$
\lim _{r \rightarrow 1} \phi\left[f\left(r e^{i \theta}\right)\right]=\phi^{*}\left[f^{*}\left(e^{i \theta}\right)\right], \quad\left|\phi^{*}\left[f^{*}\left(e^{i \theta}\right)\right]\right|=1,
$$

for almost all values of $\theta$ in the interval $\alpha_{1}<\theta<\alpha_{2}$; that is, for almost all 
points of the $\operatorname{arc} A$. Furthermore, by Lindelöf's theorem, if $\Pi_{0}$ is the point $e^{i \tau_{0}}$, then

$$
\lim _{\sigma \rightarrow 1}\left|\psi\left(\sigma e^{i \tau_{0}}\right)\right|=1 \text {. }
$$

Thus we see that if $e^{i \theta_{0}}$ is a point for which $\left|f^{*}\left(e^{i \theta_{0}}\right)\right|=1$, there corresponds to it a point $e^{i r_{0}}=\phi^{*}\left[f^{*}\left(e^{i \theta_{0}}\right)\right]$ such that $\left|\psi^{*}\left(e^{i r_{0}}\right)\right|=1$. Consider now the set $E$ of all those points $e^{i \theta}$ for which (20.3) holds. Then, by Nevanlinna's theorem, stated in $\S 8$, the set $E^{\prime}$ of points

$$
e^{i \tau}=\phi^{*}\left[f^{*}\left(e^{i \theta}\right)\right],
$$

where $e^{i \theta}$ describes the set $E$, is of measure $2 \pi$. Hence, the relation

$$
\left|\psi^{*}\left(e^{i r}\right)\right|=1
$$

is satisfied in almost all points of the circumference $|t|=1$. This, however, contradicts the fact that the relations (20.2) hold for a set of positive measure on the circumference $|t|=1$. Thus, our assumption that for some function $s(\rho)$ the $s$-measure of the set $S$ is positive has led to a contradiction. This proves the theorem.

As an immediate corollary of Theorem 14, we state the following extension of Schwarz's reflection principle:

Let $w=f(z)$ be a bounded analytic function in the unit circle $|z|<1$ :

$$
|f(z)|<1 \text {. }
$$

Let $A: \alpha_{1}<\theta<\alpha_{2}, r=1$, be an arc of the circumference $|z|=1$ such that

$$
\lim _{r \rightarrow 1} f\left(r e^{i \theta}\right)=f^{*}\left(e^{i \theta}\right), \quad\left|f^{*}\left(e^{i \theta}\right)\right|=1,
$$

for almost all values of $\theta$ in the interval $\alpha_{1}<\theta<\alpha_{2}$. Let $s(\rho)$ be a positive, continuous, monotonically increasing function for $\rho>0$ such that the integral

$$
\int_{0}^{k} \frac{s(\rho)}{\rho} d \rho
$$

is finite for some $k$. If $f(z)$ does not assume in the unit circle $|z|<1$ values which form a set of positive s-measure in the circle $|w|<1$, then $f(z)$ may be continued analytically beyond the arc $A$ in accordance with the functional relation

HARVARD UNIVERSITY,

$$
f\left(\frac{1}{\bar{z}}\right)=\frac{1}{\overline{f(z)}} \text {. }
$$

Cambridge, Mass. 